\title{
Trajectories of verbal episodic memory in middle-aged and old adults: Evidence from the English Longitudinal Study of Ageing
}

Beatriz Olaya $^{1,2}, \mathrm{MSc}, \mathrm{PhD}$, Martin Bobak ${ }^{3}, \mathrm{MD}, \mathrm{MSc}, \mathrm{PhD}$, Josep Maria Haro ${ }^{1,2}, \mathrm{MD}$, $\mathrm{PhD}, \&$ Panayotes Demakakos ${ }^{3}, \mathrm{MSc}, \mathrm{PhD}$

\footnotetext{
${ }^{1}$ Research, Innovation and Teaching Unit, Parc Sanitari Sant Joan de Déu, Universitat de Barcelona, Fundació Sant Joan de Déu, Sant Boi de Llobregat, Barcelona, Spain. ${ }^{2}$ Instituto de Salud Carlos III, Centro de Investigación Biomédica en Red de Salud Mental (CIBERSAM), Madrid, Spain.

${ }^{3}$ Department of Epidemiology and Public Health, University College London, London, UK
}

Corresponding author:

Beatriz Olaya, $\mathrm{PhD}$

Research and Development Unit, Parc Sanitari, Sant Joan de Déu

Dr. Antoni Pujadas, 42, 08830 Sant Boi de Llobregat, Barcelona, Spain

E-mail: beatriz.olaya@pssjd.org

Phone: (+34) 9355696 77; Fax: (+34) 936520051

Financial disclosure: The English Longitudinal Study of Ageing is supported by the National Institute on Aging (Grants 2RO1AG7644-01A1 and 2RO1AG017644) and a consortium of United Kingdom government departments (i.e., the Department for Education and Skills; Department for Environment, Food, and Rural Affairs;

Department of Health; Department of Trade and Industry; Department for Work and Pensions; HM Treasury Inland Revenue; the Office of the Deputy Prime Minister; and the Office for National Statistics, coordinated by the Office for National Statistics). This work has been also supported by ATHLOS Project, an European Union Horizon 2020 Research and Innovation Programme under grant agreement number 635316. Beatriz Olaya's work is supported by the Sara Borrell postdoctoral programme (CD12/00429) from the Instituto de Salud Carlos III (Spain). The funders had no role in study design, data collection and analysis, decision to publish, or preparation of the manuscript. 
Abstract word count $=275$

Main text word count $=3479$

$\mathrm{N}^{\mathrm{o}}$ figures $=1$ (2 as Supplementary figures)

$\mathrm{N}^{\mathrm{o}}$ tables $=4$

Keywords= verbal episodic memory, trajectories, longitudinal, community-dwelling, risk factors.

Brief running tittle: Trajectories of verbal episodic memory 


\section{ABSTRACT}

OBJECTIVES: To identify distinct latent groups of baseline levels and age-related decline in verbal episodic memory in middle-aged and older adults, and to identify factors associated with these trajectories.

DESIGN: Longitudinal study of 6 data collections over a period of 10 years.

SETTING: Population-based cohort in England.

PARTICIPANTS: 9,515 community-dwelling adults aged 50-79 years.

MEASUREMENTS: Six repeated measurements of immediate and delayed recall of 10 words over 10-year follow-up. Group-based trajectory modelling was used to identify patterns of baseline levels and subsequent decline in memory in two age categories (5064 and 65-79 years), and to investigate associations of trajectories with baseline predictors of group membership (gender, education, household wealth, marital status, smoking and physical activity) and time-varying covariates (depressive symptoms and number of chronic conditions).

RESULTS: Four trajectories were identified and labelled according to baseline status and decline in memory: "very low/decline" (9.8\%), "low/stable" (40.2\%), "average/stable" $(39.5 \%)$ and "good/stable" $(10.5 \%)$ in the younger group, and "very low/rapid decline" (15.7\%), "low/decline" (32.0\%), "average/stable" (38.8\%) and "good/stable" (13.5\%) among older participants. In people with stable or declining trajectories, a higher number of depressive symptoms and the presence of cardiovascular diseases were associated with worse memory. Female sex, younger age, higher education, wealth and physical activity were consistently associated with more favourable trajectories.

CONCLUSIONS: We identified four memory trajectories. Factors known to be associated with cognitive reserve (such as education, wealth and physical activity) were associated with better memory function while depressive symptoms and cardiovascular 
disease were associated with poorer memory. This suggests that interventions to reduce depressive symptoms and better manage cardiovascular risk factors and disease in midlife may help to prevent or delay future memory decline. 


\section{INTRODUCTION}

The normal ageing process is linked to cognitive decline, with dementia at the extreme end of the spectrum ${ }^{1}$. Describing and characterising cognitive decline is of special interest, as it places an immense burden on older adults, their families, and society in general ${ }^{2}$.

Longitudinal studies have consistently shown that age-related memory decline follows a curvilinear shape, ${ }^{3-5}$ and that it is around the age of 60 when cognitive decline is more likely to begin. They also found that individual trajectories varied around the mean population trajectory in terms of both starting levels and rates of change ${ }^{3,5-7}$, indicating considerable heterogeneity. Analyses of latent classes of persons who follow similar cognitive function trajectories confirmed the heterogeneity within the general population ${ }^{8-11}$. Two ${ }^{8}$, three ${ }^{9}$ and four ${ }^{10}$ different cognition trajectories have been described, distinguishing people with low starting performance and rapid decline from those with better baseline performance and a stable trajectory. Similar heterogeneity has also been observed in clinical samples of patients with Mild Cognitive Impairment ${ }^{12,13}$ and Alzheimer's Disease (AD) ${ }^{14,15}$.

The majority of previous studies focused on old or very old people ${ }^{9,13,16}$. Less is known about what is happening in the earlier years, for instance in people aged 50 to 65 , while it is at these earlier ages when preventive programmes could be more effective in reverting or ameliorating cognitive deterioration.

The first objective of this study was to identify clusters of individuals who follow distinct trajectories of verbal episodic memory within a large cohort of persons aged 50 to 79 years over a 10-year follow up. The second aim was to investigate the influences of time-varying covariates on memory within the clusters (trajectories). Finally, we investigated potential baseline predictors of trajectory membership. 
Analyses were conducted separately for younger and older participants to determine whether cognitive trajectories differed between them and whether baseline predictors of group membership or time-varying factors are more important at younger ages and may thus be a potential target for preventive programmes.

\section{METHODS}

\section{Study Population}

We used data from the English Longitudinal Study of Ageing (ELSA), a longitudinal, nationally representative survey of community-dwelling people aged 50 and older in England. Participants were recruited from households using a multi-staged stratified random probability design ${ }^{17}$. The cohort was first assessed in 2002-03 and subsequent follow-up interviews took place approximately every 2 years. Ethical approval was obtained from the Multicentre Research and Ethics Committee and participants gave informed consent.

Data from cohort members who completed a non-proxy interview and were aged 50 to 79 years old at baseline were used $(n=10,026)$. Participants who were diagnosed by a doctor with dementia (including AD) at baseline were excluded $(n=82)$. Persons who had at least one missing value in immediate and/or delayed recall at baseline or in some of the baseline covariates used for the analysis were also excluded (4.31\%), resulting in a final $n$ of 9,515 subjects.

\section{Measures}

\section{Outcomes}

Verbal episodic memory was assessed by word recall test. Participants listened to a list of 10 common words and were asked to recall as many words as possible, both immediately and after a short delay (during which other tests were performed). There were four alternative forms, so that different lists could be given in distinct waves. The 
number of words was added to obtain a total score (from 0 to 20), with higher scores indicating better memory function.

\section{Baseline covariates}

Socio-demographic characteristics at baseline included age, gender, marital status (never married, legally separated or divorced, married/remarried, and widowed), education (A-level or above recorded as "high"; O-level/Secondary education recorded as "medium" level; and no qualifications recorded as "low" education), five quintiles of household wealth (including savings and investments, value of any property or business assets, net of debt, excluding pension assets), smoking status (never smoked, ex-smokers, and current smokers) and physical activity on a weekly basis (not at all, mild, moderate and vigorous).

\section{Time-varying covariates}

At each wave, participants' self-reported medical diagnosis of cardiovascular diseases (blood pressure, heart attack or congestive heart failure, stroke, diabetes) and other chronic conditions (chronic lung disease, asthma, arthritis, osteoporosis, and cancer). The number of conditions at each wave was summed up, categorised as 0,1 , and two or more.

Depressive symptoms were measured with the 8-item Centre for Epidemiologic Studies Depression Scale $\left(\mathrm{CES}-\mathrm{D}^{18}\right)$. The response format was binary (Yes/No). A total score was calculated (from 0 to 8), with higher scores indicating greater severity.

\section{Statistical Analysis}

Group-based trajectory models (GBTM) ${ }^{19}$ were calculated separately for people aged 50-64 and 65-79 years old at baseline. This method fits a semi-parametric mixture model to longitudinal data using a maximum-likelihood method. The time metric was 
years into the study (0-10 years). The outcome was memory scores assessed in waves 16, and modelled with a censored normal distribution using Stata Traj plug-in ${ }^{20}$.

The number and shape (via polynomial functions) of trajectories were determined by analysing 2-5 group models without covariates. We decided on the final number of trajectories using likelihood criteria such as BIC, while trying to be as parsimonious as possible ${ }^{19,21}$. Average posterior probabilities above $70 \%$ also indicate optimal fit ${ }^{19}$.

Covariates were simultaneously introduced in the model using two model extensions ${ }^{20}$ to determine: 1 ) how events that occur during the follow-up (time-varying) affect the trajectories and 2) whether they predict group membership (covariates at baseline). Time-varying covariates (depression, number of CVD and non CVD) were included simultaneously with time. Adjustment for time-varying covariates can alter the degree and rate of change within each trajectory ${ }^{19}$, therefore trajectories were presented after adjusting for these covariates. In the second part of the model, the probabilities of trajectory group membership (derived after including time and time-varying covariates) are treated as the dependent variable predicted by covariates assessed at baseline in a fashion similar to a multinomial analysis ${ }^{17}$. Since the parameters defining the trajectories and the probabilities of group membership are jointly estimated, group assignments based on the highest posterior probabilities are not used, thus reducing error assignment ${ }^{19}$. Variables with multiple categories (education, marital status and smoking status) were introduced as dummy variables.

Missing data were handled with a maximum likelihood approach based on a missing-at-random assumption ${ }^{21}$. In order to explore differential attrition rates across trajectory groups and whether these differences could affect the main results, an extension of GBTM that accounts for non-random attrition was used ${ }^{22}$. All analyses 
were performed with Stata software, version 12.1 (Stata Corp LP, College Station, Texas, USA). A two-side $p$ value of less than 0.05 was considered as statistically significant.

\section{RESULTS}

A total of 4,238 persons (44.5\%) had completed information on the outcome across all waves while $17 \%(n=1,573)$ of participants had died by the end of the study. Tables 1 and 2 summarise the baseline characteristics of people $(N=9,515)$ and the distribution of the outcome and time-varying covariates across waves.

A 4-group model produced the best BIC values in both age groups. The average posterior probabilities were all above 0.70 , indicating good fit. The four trajectory models were re-estimated including time-varying covariates and the shape and probability of trajectory groups were similar to those identified without such an adjustment.

Trajectory groups were labelled according to baseline memory scores and decline (Figure 1). In the younger group, 9.8\% ("very low/decline") presented a negative linear term and a very low memory score at baseline (Table 3). The second group (40.2\%) had low baseline score and a stable trajectory ("low/stable"). The third (39.5\%) and fourth $(10.5 \%)$ presented also stable trajectories and were labelled as "average/stable" and "good/stable", respectively.

In the older cohort, $15.7 \%$ had very poor scores at baseline and rapid cognitive decline ("very low/rapid decline") and 32\% had poor baseline memory and moderate decline ("low/decline"). The "average/stable" (38.8\%) and the "good/stable" (13.5\%) classes showed stability in memory function over time.

Table 3 displays the estimated coefficients for time and time-varying covariates within each group. In the younger cohort and at a given trajectory time point, each unit increase in depressive symptoms was associated with lower levels of memory in the 
"very low/decline", "low/stable" and "average/stable" groups but did not affect memory among people in the "good/stable" group. In the older cohort, each increase in depressive symptoms was related to worse memory function in the "low/decline", "average/stable" and "good/stable" but not in the "very low/rapid decline" group.

Each unit increase in the number of CVDs negatively affected the memory of middle-aged people belonging to the "very low/decline", "low/stable" and "average/stable" groups; in the older cohort, an association was only found in the "low/ decline" group. Similarly, each unit increase in the number of other chronic conditions was associated with lower memory scores in the "low/stable" trajectory of the middleaged category, while a significant improvement was observed in the "low/ decline" and in the "average/stable" older cohort groups.

Table 4 displays ORs of group membership by baseline predictors. In the younger cohort, higher levels of wealth, medium or high level of education (compared with low), female sex and younger age were associated with increased odds of membership of the three stable trajectories, relative to the "very low/decline" group. Higher levels of physical activity predicted the "average/stable" and "good/stable" trajectories. Being married or separated/divorced at baseline predicted the "low/stable" trajectory, compared with the "very low/decline" group. Current smokers were less likely to be in the "low/stable" group, compared with the "very low/decline" $(\mathrm{OR}=0.92$, $\mathrm{CI} 95 \%=0.89-0.95)$.

In the older category, higher levels of wealth, younger age and greater physical activity were significant predictors of the three more favourable trajectories, compared with the "very low/rapid decline" trajectory. Women were more likely than men to follow an "average/stable" $(\mathrm{OR}=2.21, \mathrm{CI} 95 \%=1.51-2.87)$ and "good/stable" $(\mathrm{OR}=4.62$, CI95\%=2.88-6.36) trajectory and smokers at baseline were less likely than non-smokers 
to follow an "average/stable" trajectory, compared with the "very low/rapid decline" $(\mathrm{OR}=0.67, \mathrm{CI} 95 \%=0.40-0.94)$.

\section{Probability of drop-out}

The probability of attrition was higher for the "very low/decline" and "very low/rapid decline" trajectories in the youngest and oldest categories, respectively (Supplementary Figure S1). However, trajectories in the models with and without the attrition extension and the probabilities of belonging to each trajectory group were similar (Figure 1 and Supplementary Figure S2).

\section{DISCUSSION}

Our findings suggest a presence of four distinct trajectories of verbal episodic memory in both age groups, although the shape and probabilities were different; in the younger cohort, three out of four latent groups showed a stable memory function over ten years whereas among older adults, memory decline is observed for two of the four trajectories.

Overall, these results confirm the heterogeneity in cognitive ageing reported by previous studies ${ }^{23,24}$ and are consistent with research into latent groups of cognitive decline ${ }^{8,11}$ suggesting that rapid cognitive decline is not observed in a proportion of older people. However, our findings suggest that this proportion depends on age. Only about $10 \%$ of middle-aged people (50- 64 years) had some degree of memory decline whereas $48 \%$ of older people (65-79 years) showed a memory decline. This pattern indicates that interventions should be delivered at early stages, probably when people are in their $50 \mathrm{~s}^{25}$.

Post-mortem and neuroimaging studies in community-based samples have previously shown that persons with rapid cognitive decline and low performance were more likely to present underlying neuropathology and low hippocampal volume $\mathrm{e}^{8,11}$. Participants in our study belonging to the "very low/decline" or "very low/rapid 
decline" might therefore present some degree of neuropathology associated with AD and dementia ${ }^{13}$, although in our study people with a diagnosis of dementia at baseline were excluded. Interventions to prevent progression to AD or other dementias should be delivered before disease symptoms are manifested ${ }^{26}$.

Our results suggest that depressive symptomatology can negatively affect the memory of people who have stable trajectories. Depression has previously been associated with worse performance in cognitive tests in population-based samples of older adults ${ }^{27}$. However, in our data depressive symptoms were not associated with memory decline in the older cohort, suggesting that at this age cognitive decline may depend on other underlying pathologies rather than depression ${ }^{28}$.

Presence of CVD was also associated with lower memory scores in all trajectory groups of those aged 50-64 years. However, memory appears to be not affected by CVDs among the oldest cohort. This is consistent with previous studies showing that cardiovascular risk factors and CVD are related to cognitive decline in midlife ${ }^{29,30}$. CVD may contribute to subtle brain damage at early stages that becomes more apparent at older ages ${ }^{31}$. Thus, management of CVD in midlife could be an effective way to prevent future cognitive deterioration, regardless of the level of cognitive performance. Presence of non-cardiovascular chronic conditions was only associated with lower memory in the "low/stable" trajectory for the younger cohort. This is in line with previous studies reporting an association between conditions such as musculoskeletal diseases, lung diseases or arthritis, and cognitive decline ${ }^{32}$. Conversely, in two older adult trajectory groups, an improvement in memory function was associated with an increase in the number of chronic conditions. The reasons for this are unclear and further epidemiological studies are needed to confirm our findings. 
Results from baseline predictors of trajectory membership show that women are more likely to follow a stable trajectory with a good memory function independently of other confounders (e.g., education). This might be explained by genuine differences in brain structures and the role of sex hormones affecting hippocampal structures involved in the episodic memory ${ }^{33}$, but may also reflect gender differences in behavioural and biological risk factors. In keeping with previous literature, people with low educational attainment were more likely to show a rapid decline in memory function. Education might have positive and profound effects on brain structures during the early stages of $\operatorname{life}^{34}$, contributing to increased cognitive reserve ${ }^{35,36}$. Cognitive reserve might help the brain to compensate for the presence of neuropathology and delay the onset of clinical symptoms. Wealth was also related to better trajectories of memory function, independently from education. Wealth might be related to intellectually-demanding occupations, stimulating environments or better access to health systems with a positive impact on cognitive function ${ }^{37}$.

Physical activity is an important predictor of trajectories in midlife and older ages, and it increases the probability of being in stable groups. The literature suggests that physical activity is a powerful protective factor and constitutes part of the cognitive reserve ${ }^{35}$. However, people in the unfavourable trajectory groups (i.e., poor cognitive performance and rapid decline) might present a lack of mobility and high levels of disability as a consequence of their cognitive status or of an underlying neuropathology.

Marital status predicted being in the "low/stable" group only among middleaged people. Other studies have shown that being single, compared with being married, is associated with poor cognitive function ${ }^{38}$ and faster rates of cognitive deterioration ${ }^{16}$. A spouse could be an important source of emotional and social support ${ }^{39}$, and thus offer protection against cognitive deterioration in later life. Smoking at baseline significantly 
predicted a poor memory trajectory, which is consistent with literature showing that smoking is a risk factor for cognitive decline ${ }^{40}$.

Our findings should be considered in the light of limitations. First, people with a self-reported diagnosis of dementia were excluded from the analysis but we cannot rule out the possibility that persons with a current diagnosis were finally included in the sample. There is evidence that repeated memory tests might result in improved performance due to familiarity with the task ${ }^{41}$ and that the highest improvements are particularly evident at first re-assessments but diminish with subsequent waves ${ }^{42}$. Statistical strategies to account for the practice effect may affect the estimated rates of cognitive change but not the estimated association of risk factors with change ${ }^{42}$. The use of alternative list of words in our study could help minimise the practice effect ${ }^{43}$. Moreover, it is unlikely that the practice effect influenced the separation of study subjects into trajectory groups. Non-ignorable drop-out was addressed by using a modelling extension designed to alleviate bias in the estimation of group membership probabilities $^{22}$. The shape of trajectories and size of latent groups were similar when using models with and without this extension, suggesting that attrition bias only minimally affected our results. It is difficult to confirm the equivalence between groups in distinct age categories. For example, people belonging to the "very low/decline" group in the middle-aged group probably have poorer cognition than older adults in the "very low" group. Finally, trajectories of other cognitive domains (e.g., working memory) may have different patterns.

Our findings suggest that there is substantial heterogeneity in how episodic memory evolves over time and identifies four episodic memory trajectories. Second, memory deterioration is not restricted to older adults; a modest decline in memory can be observed as early as midlife. Third, a subgroup of older adults can maintain optimal 
memory function, possibly due to a good cognitive reserve and health status. And fourth, risk factors such as depressive symptoms and cardiovascular diseases were strongly associated with lower memory function not only in persons with rapid decline, but also in those with optimal memory trajectories. Early interventions (e.g., at the age of 50) should be targeted to ameliorate the decline observed among persons with poor memory function and rapid deterioration. Targeting depressive symptoms and cardiovascular diseases, regardless of age and level of cognition, might help prevent memory decline, and both middle-aged and older adults might benefit from programmes promoting healthy lifestyles. 


\section{ACKNOWLEDGEMENTS}

The authors would like to thank Maria Victoria Moneta for her comments and advice on the statistical section.

Conflict of interest: The authors declare no conflict of interest.

Funding sources: The English Longitudinal Study of Ageing is supported by the National Institute on Aging (Grants 2RO1AG7644-01A1 and 2RO1AG017644) and a consortium of United Kingdom government departments (i.e., the Department for Education and Skills; Department for Environment, Food, and Rural Affairs; Department of Health; Department of Trade and Industry; Department for Work and Pensions; the HM Treasury Inland Revenue; the Office of the Deputy Prime Minister; and the Office for National Statistics, coordinated by the Office for National Statistics). This work has been also supported by ATHLOS Project, a European Union Horizon 2020 Research and Innovation Programme under grant agreement number 635316. Beatriz Olaya's work is supported by the Sara Borrell postdoctoral programme (CD12/00429) from the Instituto de Salud Carlos III (Spain). The funders had no role in study design, data collection and analysis, decision to publish, or preparation of the manuscript.

Author contributions: B.O conducted statistical analyses and wrote the main body of the manuscript; P.D. provided the data, commented on intellectual content and approved the final version to be published; M.B and J.M.H. critically revised the paper and approved the final version to be published. 


\section{REFERENCES}

1. Plassman BL, Williams JW, Burke JR, Holsinger T, Benjamin S. Systematic review: factors associated with risk for and possible prevention of cognitive decline in later life. Ann Intern Med. 2010;153(3):182-193. doi:10.7326/00034819-153-3-201008030-00258.

2. Chodosh J, Miller-Martinez D, Aneshensel CS, Wight RG, Karlamangla AS. Depressive Symptoms, Chronic Diseases, and Physical Disabilities as Predictors of Cognitive Functioning Trajectories in Older Americans. J Am Geriatr Soc. 2010;58(12):2350-2357. doi:10.1111/j.1532-5415.2010.03171.x.

3. Tampubolon G. Cognitive Ageing in Great Britain in the New Century: Cohort Differences in Episodic Memory. PLoS One. 2015;10(12):e0144907. doi:10.1371/journal.pone.0144907.

4. Rönnlund M, Nyberg L, Bäckman L, Nilsson L-G. Stability, growth, and decline in adult life span development of declarative memory: cross-sectional and longitudinal data from a population-based study. Psychol Aging. 2005;20(1):318. doi:10.1037/0882-7974.20.1.3.

5. Nyberg L, Lövdén M, Riklund K, Lindenberger U, Bäckman L. Memory aging and brain maintenance. Trends Cogn Sci. 2012;16(5):292-305. doi:10.1016/j.tics.2012.04.005.

6. Rabbitt P, Diggle P, Holland F, McInnes L. Practice and drop-out effects during a 17-year longitudinal study of cognitive aging. J Gerontol B Psychol Sci Soc Sci. 2004;59(2):P84-97.

7. Rabbitt P, Osman P, Moore B, Stollery B. There are stable individual differences in performance variability, both from moment to moment and from day to day. $Q$ J Exp Psychol A. 2001;54(4):981-1003. doi:10.1080/713756013.

8. Zahodne LB, Wall MM, Schupf N, et al. Late-life memory trajectories in relation to incident dementia and regional brain atrophy. J Neurol. 2015.

doi:10.1007/s00415-015-7871-8.

9. Terrera GM, Brayne C, Matthews F. One size fits all? Why we need more sophisticated analytical methods in the explanation of trajectories of cognition in older age and their potential risk factors. Int Psychogeriatr. 2010;22(2):291-299. doi:10.1017/S1041610209990937.

10. Marioni RE, Proust-Lima C, Amieva H, et al. Cognitive lifestyle jointly predicts longitudinal cognitive decline and mortality risk. Eur J Epidemiol. 2014;29(3):211-219. doi:10.1007/s10654-014-9881-8.

11. Hayden KM, Reed BR, Manly JJ, et al. Cognitive decline in the elderly: An analysis of population heterogeneity. Age Ageing. 2011;40(6):684-689. doi:10.1093/ageing/afr101.

12. Xie H, Mayo N, Koski L. Identifying and characterizing trajectories of cognitive change in older persons with mild cognitive impairment. Dement Geriatr Cogn Disord. 2011;31(2):165-172. doi:10.1159/000323568.

13. Mungas D, Beckett L, Harvey D, et al. NIH Public Access. 2011;25(3):606-619. doi:10.1037/a0019502.Heterogeneity of cognitive trajectories in diverse older 
person. Psychol Aging. 2011;25:606-619.

14. Wilson RS, Segawa E, Boyle PA, Anagnos SE, Hizel LP, Bennett DA. The Natural History of Cognitive Decline in Alzheimer's Disease. Psychol Aging. 2012;27(4):1008-1017. doi:10.1037/a0029857.

15. Bilgel M, An Y, Lang A, et al. Trajectories of Alzheimer disease-related cognitive measures in a longitudinal sample. Alzheimer's Dement.

2014;10(6):735-742. doi:10.1016/j.jalz.2014.04.520.

16. Karlamangla AS, Miller-Martinez D, Aneshensel CS, Seeman TE, Wight RG, Chodosh J. Trajectories of cognitive function in late life in the United States: Demographic and socioeconomic predictors. Am J Epidemiol. 2009;170(3):331342. doi:10.1093/aje/kwp154.

17. Steptoe A, Breeze E, Banks J, Nazroo J. Cohort profile: the English longitudinal study of ageing. Int J Epidemiol. 2013;42(6):1640-1648. doi:10.1093/ije/dys168.

18. Wallace RB, Regula A, Mary H, et al. HRS/AHEAD Documentation Report Documentation of Affective Functioning Measures in the Health and Retirement Study. 2000. Ann Arbor: Survey Research Center, University of Michigan; 2000.

19. Nagin DS. Group-Based Modeling of Development. Cambridge, MA: Harvard University Press; 2005.

20. Jones BL, Nagin DS. A Note on a Stata Plugin for Estimating Group-based Trajectory Models. Sociol Methods Res. 2013;42(4):608-613. doi:10.1177/0049124113503141.

21. Nagin DS, Odgers CL. Group-based trajectory modeling in clinical research. Annu Rev Clin Psychol. 2010;6:109-138. doi:10.1146/annurev.clinpsy.121208.131413.

22. Haviland a. M, Jones BL, Nagin DS. Group-based Trajectory Modeling Extended to Account for Nonrandom Participant Attrition. Sociol Methods Res. 2011;40(2):367-390. doi:10.1177/0049124111400041.

23. Wilson RS, Li Y, Bienias L, Bennett DA. Cognitive decline in old age: Separating retest effects from the effects of growing older. Psychol Aging. 2006;21:774-789.

24. Wilson RS, Beckett LA, Barnes LL, et al. Individual differences in rates of change in cognitive abilities of older persons. Psychol Aging. 2002;17:179-193.

25. Salthouse TA. When does age-related cognitive decline begin? Neurobiol Aging. 2009;30(4):507-514. doi:10.1016/j.neurobiolaging.2008.09.023.

26. Emery VOB. Alzheimer disease: are we intervening too late? Pro. J Neural Transm. 2011;118(9):1361-1378. doi:10.1007/s00702-011-0663-0.

27. Ganguli M. Depression, cognitive impairment and dementia: Why should clinicians care about the web of causation? Indian J Psychiatry. 2009;51 Suppl 1(January 2009):S29-34.

28. Ganguli M, Du Y, Dodge HH, Ratcliff GG, Chang C-CH. Depressive symptoms and cognitive decline in late life: a prospective epidemiological study. Arch Gen Psychiatry. 2006;63(2):153-160. doi:10.1001/archpsyc.63.2.153. 
29. Anstey KJ, Sargent-Cox K, Garde E, Cherbuin N, Butterworth P. Cognitive development over 8 years in midlife and its association with cardiovascular risk factors. Neuropsychology. 2014;28(4):653-665. doi:10.1037/neu0000044.

30. Debette S, Seshadri S, Beiser A, et al. Midlife vascular risk factor exposure accelerates structural brain aging and cognitive decline. Neurology. 2011;77(5):461-468. doi:10.1212/WNL.0b013e318227b227.

31. Launer LJ. The epidemiologic study of dementia: a life-long quest? Neurobiol Aging. 2005;26(3):335-340. doi:10.1016/j.neurobiolaging.2004.03.016.

32. Caracciolo B, Gatz M, Xu W, Marengoni A, Pedersen NL, Fratiglioni L. Relationship of subjective cognitive impairment and cognitive impairment no dementia to chronic disease and multimorbidity in a nation-wide twin study. $J$ Alzheimers Dis. 2013;36(2):275-284. doi:10.3233/JAD-122050.

33. Li R, Singh M. Sex differences in cognitive impairment and Alzheimer's disease. Front Neuroendocrinol. 2014;35(3):385-403. doi:10.1016/j.yfrne.2014.01.002.

34. Albert MS. How does education affect cognitive function? Ann Epidemiol. 1995;5(1):76-78. h

35. Stern Y. Cognitive reserve in ageing and Alzheimer's disease. Lancet Neurol. 2012;11(11):1006-1012. doi:10.1016/S1474-4422(12)70191-6.

36. Ganguli M. Depression, cognitive impairment and dementia: Why should clinicians care about the web of causation? Indian J Psychiatry. 2009;51 Suppl 1:S29-34.

37. Koster A, Penninx BWJH, Bosma H, et al. Socioeconomic differences in cognitive decline and the role of biomedical factors. Ann Epidemiol. 2005;15(8):564-571. doi:10.1016/j.annepidem.2005.02.008.

38. Yeh S-CJ, Liu Y-Y. Influence of social support on cognitive function in the elderly. BMC Health Serv Res. 2003;3(1):9. doi:10.1186/1472-6963-3-9.

39. Seeman TE, Berkman LF. Structural characteristics of social networks and their relationship with social support in the elderly: who provides support. Soc Sci Med. 1988;26(7):737-749.

40. Baumgart M, Snyder HM, Carrillo MC, Fazio S, Kim H, Johns H. Summary of the evidence on modifiable risk factors for cognitive decline and dementia: A population-based perspective. Alzheimers Dement. 2015;11(6):718-726. doi:10.1016/j.jalz.2015.05.016.

41. Zehnder AE, Bläsi S, Berres M, Spiegel R, Monsch AU. Lack of practice effects on neuropsychological tests as early cognitive markers of Alzheimer disease? Am J Alzheimers Dis Other Demen. 22(5):416-426. doi:10.1177/1533317507302448.

42. Vivot A, Power MC, Glymour MM, et al. Jump, Hop, or Skip: Modeling Practice Effects in Studies of Determinants of Cognitive Change in Older Adults. Am J Epidemiol. 2016;183(4):302-314. doi:10.1093/aje/kwv212.

43. Beglinger LJ, Gaydos B, Tangphao-Daniels O, et al. Practice effects and the use of alternate forms in serial neuropsychological testing. Arch Clin Neuropsychol. 2005;20(4):517-529. doi:10.1016/j.acn.2004.12.003. 
Table 1 . Baseline characteristics of the sample by age category $(N=9,515)$

\begin{tabular}{|c|c|c|c|}
\hline & $50-64$ & $65-78$ & \\
\hline & $n=5523$ & $n=3992$ & $P$ value $^{\mathrm{a}}$ \\
\hline Drop-outs at Wave 6, n (\%) & $2144(38.8)$ & $2164(54.2)$ & $<0.001$ \\
\hline Female, n (\%) & $2951(53.4)$ & $2154(54)$ & 0.611 \\
\hline Marital status, n (\%) & & & $<0.001$ \\
\hline Single & $318(5.8)$ & $211(5.3)$ & \\
\hline Married/remarried & $4132(74.8)$ & $2552(63.9)$ & \\
\hline Separated/divorced & $788(14.3)$ & $314(7.8)$ & \\
\hline Widowed & $285(5.2)$ & $915(22.9)$ & \\
\hline Education, n (\%) & & & $<0.001$ \\
\hline Low & $1740(31.5)$ & $2036(51)$ & \\
\hline Medium & $1790(32.4)$ & $1115(27.9)$ & \\
\hline High & $1993(36.1)$ & $841(21.1)$ & \\
\hline Quintiles of wealth, n (\%) & & & $<0.001$ \\
\hline Lowest & $816(14.8)$ & $825(20.7)$ & \\
\hline $2^{\text {nd }}$ & $1066(19.3)$ & $816(20.4)$ & \\
\hline $3^{r d}$ & 1139 (20.6) & $811(20.3)$ & \\
\hline $4^{\text {th }}$ & 1187 (21.5) & 795 (19.9) & \\
\hline Highest & $1315(23.8)$ & 745 (18.7) & 0.012 \\
\hline Physical Activity, n (\%) & & & $<0.001$ \\
\hline No $P A$ & $349(6.3)$ & $442(4.1)$ & \\
\hline Mild PA & $624(11.3)$ & $638(16.0)$ & \\
\hline Moderate PA & $2677(48.5)$ & $1973(49.4)$ & \\
\hline Vigorous $P A$ & $1873(33.9)$ & $939(23.1)$ & \\
\hline Smoking status, n (\%) & & & $<0,001$ \\
\hline Neversmoked & $1977(35.8)$ & $1379(34.5)$ & \\
\hline Ex-smoker & $2291(41.5)$ & $2032(50.9)$ & \\
\hline Currentsmoker & $1255(22.7)$ & $581(14.5)$ & \\
\hline
\end{tabular}

Note: $\mathrm{SD}=$ Standard Deviation; $\mathrm{PA}=$ Physical Activity. Low education level included people with no qualifications.

${ }^{\mathrm{a} C h i}$-square and one-way ANOVA tests were performed to determine differences in baseline characteristics between age groups. 
Table 2. Distribution of the outcome (episodic memory score) and time-varying covariates

\begin{tabular}{|c|c|c|c|c|c|c|}
\hline & \multicolumn{6}{|c|}{ 50-64 years old } \\
\hline & $\begin{array}{c}\text { Time } 0 \\
\text { (baseline) }\end{array}$ & $\begin{array}{c}\text { Time } 1 \\
(2 \text { years })\end{array}$ & $\begin{array}{c}\text { Time } 2 \\
(4 \text { years }) \\
\end{array}$ & $\begin{array}{c}\text { Time } 3 \\
\text { (6 years) }\end{array}$ & $\begin{array}{c}\text { Time } 4 \\
(8 \text { years })\end{array}$ & $\begin{array}{c}\text { Time } 5 \\
(10 \text { years })\end{array}$ \\
\hline Episodic memory, mean (SD) & $10.67(3.15)$ & $11.11(3.11)$ & $11.25(3.25)$ & $11.17(3.24)$ & $11.12(3.36)$ & $11.22(3.37)$ \\
\hline CES-D sum, mean (SD) & $1.48(1.99)$ & $1.47(1.94)$ & $1.37(1.93)$ & $1.25(1.84)$ & $1.34(1.87)$ & $1.16(1.75)$ \\
\hline \multicolumn{7}{|l|}{ CVD, n (\%) } \\
\hline None & $3598(65.1)$ & $3192(71.3)$ & $2690(68.1)$ & $2325(64.3)$ & $2216(62.2)$ & $2088(61.9)$ \\
\hline One & $1579(28.6)$ & $1089(24.3)$ & $1181(29.9)$ & $1213(33.5)$ & $1256(35.2)$ & $1216(36.0)$ \\
\hline Two or more & $346(6.3)$ & $193(4.3)$ & 77 (1.9) & $77(2.1)$ & $93(2.6)$ & $71(2.1)$ \\
\hline \multicolumn{7}{|l|}{ Non CVD, n (\%) } \\
\hline None & $3304(59.8)$ & $2660(59.4)$ & $2259(57.2)$ & $1963(54.3)$ & $1823(51.1)$ & $1661(49.2)$ \\
\hline One & $1689(30.6)$ & $1406(31.4)$ & $1304(33.0)$ & $1254(34.7)$ & $1304(36.6)$ & $1280(37.9)$ \\
\hline Two or more & $530(9.6)$ & $408(9.1)$ & $384(9.7)$ & $398(11.0)$ & $440(12.3)$ & $436(12.9)$ \\
\hline & \multicolumn{6}{|c|}{$65-79$ years old } \\
\hline Episodic memory, mean (SD) & $8.56(3.34)$ & $8.93(3.41)$ & $8.86(3.50)$ & $8.72(3.49)$ & $8.49(3.63)$ & $8.51(3.67)$ \\
\hline CES-D sum, mean (SD) & $1.59(1.93)$ & 1.59 (1.91) & $1.55(1.94)$ & $1.51(1.91)$ & $1.68(1.97)$ & $1.48(1.87)$ \\
\hline \multicolumn{7}{|l|}{ CVD, n (\%) } \\
\hline None & $1865(46.7)$ & $1822(58.1)$ & $1461(54.9)$ & $1162(50.2)$ & 993 (47.2) & 867 (47.4) \\
\hline One & $1558(14.2)$ & $1075(34.3)$ & 1115 (41.9) & $1060(45.8)$ & 1014 (48.2) & 885 (48.4) \\
\hline Two or more & 569 (14.2) & $233(7.44)$ & $83(3.1)$ & $93(4.0)$ & $97(4.6)$ & $75(4.1)$ \\
\hline \multicolumn{7}{|l|}{ Non CVD, n (\%) } \\
\hline None & $1851(46.4)$ & $1433(45.8)$ & $1173(44.1)$ & $968(41.8)$ & $844(40.1)$ & $690(37.7)$ \\
\hline One & $1564(39.2)$ & $1268(40.5)$ & $1093(41.1)$ & $995(43.0)$ & $903(43.0)$ & $820(44.9)$ \\
\hline Two or more & $577(14.4)$ & $430(13.7)$ & $394(14.8)$ & $351(15.2)$ & $355(16.9)$ & $318(17.4)$ \\
\hline
\end{tabular}

Note: $\mathrm{CES}-\mathrm{D}=$ Center for Epidemiologic Studies Depression Scale; CVD= Number of cardiovascular diseases; non $\mathrm{CVD}=\mathrm{Number}$ of other chronic conditions. Episodic memory scores ranged from 0 to 20; CES-D scores ranged from 0 to 8 . 
Table 3.Parameter estimations for Verbal Episodic Memory trajectories (model with time-varying covariates) by age group ${ }^{a}$

\begin{tabular}{|c|c|c|}
\hline & 50-64years & 65-79years \\
\hline & Very low/decline & Very low/rapid decline \\
\hline Intercept & $7.09(0.147)^{* * *}$ & $4.66(0.174)^{* * *}$ \\
\hline Years & $-0.09(0.020)^{* * *}$ & $-0.24(0.024) * * *$ \\
\hline CES-D & $-0.16(0.032)^{* * *}$ & $-0.06(0.037)$ \\
\hline CVD & $-0.24(0.113)^{*}$ & $0.11(0.111)$ \\
\hline \multirow[t]{2}{*}{ Non-CVD } & $-0.17(0.103)$ & $0.11(0.11)$ \\
\hline & Low/stable & Low/decline \\
\hline Intercept & $9.93(0.090) * * *$ & $7.90(0.143)^{* * *}$ \\
\hline Years & $0.07(0.028)^{* *}$ & $-0.17(0.016)^{* * *}$ \\
\hline Years $^{2}$ & $-0.01(0.003)^{* *}$ & - \\
\hline CES-D & $-0.12(0.016)^{* * *}$ & $-0.14(0.026)^{* * *}$ \\
\hline CVD & $-0.18(0.056)^{* *}$ & $-0.19(0.075)^{*}$ \\
\hline \multirow[t]{2}{*}{ Non-CVD } & $-0.13(0.050)^{* *}$ & $0.28(0.073)^{* * *}$ \\
\hline & Average/stable & Average/stable \\
\hline Intercept & $12.15(0.095)^{* *}$ & $9.84(0.115)^{* * *}$ \\
\hline Years & $0.12(0.028)^{* * *}$ & $0.10(0.035)^{* *}$ \\
\hline Years $^{2}$ & $-0.01(0.003)^{* *}$ & $-0.02(0.003) * * *$ \\
\hline CES-D & $-0.05(0.017)^{* *}$ & $-0.10(0.023) * * *$ \\
\hline CVD & $-0.19(0.058)^{* *}$ & $-0.08(0.068)$ \\
\hline \multirow[t]{2}{*}{ Non-CVD } & $-0.09(0.049)$ & $0.14(0.062)^{*}$ \\
\hline & Good/stable & Good/stable \\
\hline Intercept & $14.38(0.141)^{* * *}$ & $12.53(0.153)^{* * *}$ \\
\hline Years & $0.27(0.052)^{* * *}$ & $0.16(0.055)^{* *}$ \\
\hline Years $^{2}$ & $-0.02(0.005)^{* * *}$ & $-0.02(0.005)^{* * *}$ \\
\hline CES-D & $-0.05(0.032)$ & $-0.08(0.035)^{*}$ \\
\hline CVD & $-0.17(0.116)$ & $-0.06(0.101)$ \\
\hline Non-CVD & $-0.03(0.091)$ & $0.02(0.087)$ \\
\hline
\end{tabular}

Note: Standard Errors are in parentheses. CES-D= Center for Epidemiologic Studies Depression Scale; $\mathrm{CVD}=$ Number of cardiovascular diseases (heart problems, diabetes, hypertension, stroke), 0,1 and 2 or more; Non-CVD $=$ Number of non-cardiovascular diseases (asthma, cancer, osteoporosis, arthritis, chronic lung diseases), 0, 1, and 2 or more.

${ }^{\text {a }}$ Time was measured as years into the study (from 0 to 10 years). Years since baseline, depressive symptoms, number of CVD and non CVD were simultaneously introduced into the models. Coefficient estimates for time-varying variables indicate the association of each unit of change in that particular covariate with an increase (or decrease) in the memory score, at a given trajectory point. 

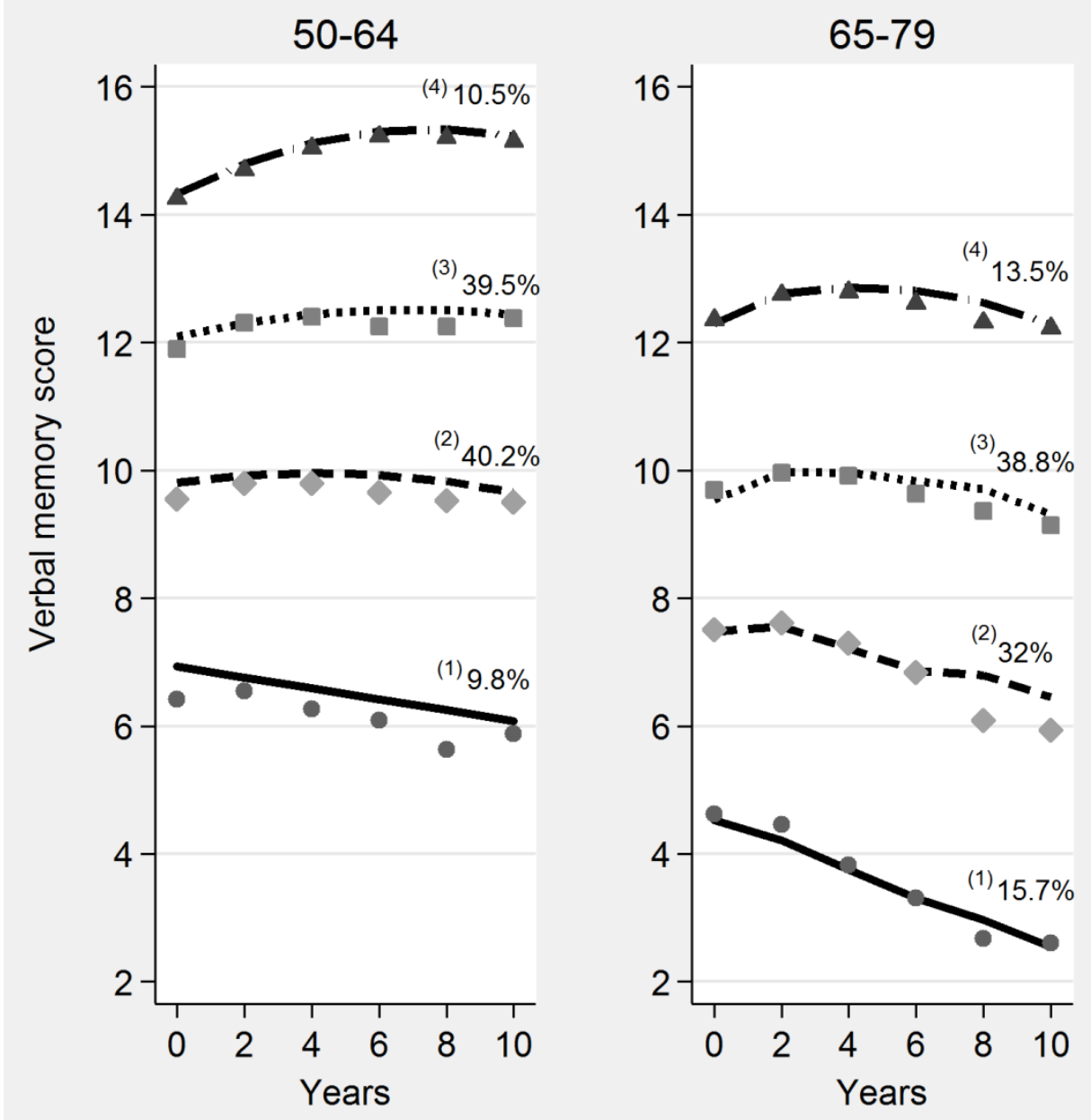

Figure 1. Trajectories of verbal episodic memory by age group

Note: "Years" indicate years since baseline. Verbal memory scores range from 0 to 20 . These trajectories were calculated with a model including time-varying covariates (depression, CVD, and other chronic conditions) and predictors of group membership at baseline (age, gender, marital status, education level, wealth, smoking status and physical activity). 
Table 4. Factors associated with trajectory group membership by age category

\begin{tabular}{|c|c|c|c|c|c|c|}
\hline \multirow[b]{2}{*}{ Variables at baseline } & \multicolumn{3}{|c|}{$50-64^{\mathrm{a}}$} & \multicolumn{3}{|c|}{$65-79^{b}$} \\
\hline & Low/stable & Average/stable & Good/stable & Low/decline & Average/stable & Good/stable \\
\hline Wealth & $1.23(1.09-1.36)^{* *}$ & $1.51(1.35-1.68)^{* * *}$ & $1.83(1.56-2.10) * * *$ & $1.19(1.05-1.34)^{* *}$ & $1.51(1.34-1.67) * * *$ & $1.68(1.44-1.93)^{* * *}$ \\
\hline \multicolumn{7}{|l|}{ Gender (ref. Males) } \\
\hline Females & $1.59(1.16-2.02)^{* *}$ & $3.53(2.54-4.52)^{* * *}$ & $8.6(5.42-11.79)^{* * *}$ & $0.98(0.67-1.29)$ & $2.21(1.51-2.87)^{* * *}$ & $4.62(2.88-6.36)^{* * *}$ \\
\hline \multicolumn{7}{|l|}{ Education level (ref. low) } \\
\hline Medium & $1.99(1.36-2.62)^{* *}$ & $4.7(3.18-6.23) * * *$ & $14.71(4.4-25.03) * *$ & $1.82(1.15-2.49)^{*}$ & $2.98(1.96-4.01)^{* * *}$ & $8.15(4.47-11.83)^{* * *}$ \\
\hline Age at baseline & $0.92(0.89-0.95)^{* * *}$ & $0.84(0.81-0.87)^{* * *}$ & $0.77(0.74-0.81)^{* * *}$ & $0.92(0.89-0.96) * * *$ & $0.81(0.78-0.84)^{* * *}$ & $0.72(0.69-0.76)^{* * *}$ \\
\hline \multicolumn{7}{|l|}{ Marital status (ref. single) } \\
\hline Married & $2.48(1.35-3.62)^{*}$ & $1.75(0.93-2.57)$ & $1.47(0.55-2.39)$ & $1.32(0.6-2.04)$ & $1.96(0.86-3.07)$ & $1.36(0.34-2.38)$ \\
\hline Separated & $2.41(1.11-3.70)^{*}$ & $1.74(0.78-2.69)$ & $1.43(0.38-2.48)$ & $1.13(0.31-1.94)$ & $2.21(0.68-3.74)$ & $1.64(0.10-3.19)$ \\
\hline Widowed & $2.92(0.95-4.88)$ & $1.52(0.44-2.59)$ & $1.64(0.08-3.20)$ & $1.37(0.58-2.16)$ & $2.18(0.88-3.47)$ & $2.31(0.48-4.14)$ \\
\hline \multicolumn{7}{|l|}{$\begin{array}{l}\text { Smoking status (ref. never } \\
\text { smoked) }\end{array}$} \\
\hline Current smoker & $0.72(0.48-0.96)^{*}$ & $0.76(0.50-1.02)$ & $0.72(0.38-1.06)$ & $0.87(0.5-1.25)$ & $0.67(0.4-0.94)^{*}$ & $0.86(0.41-1.31)$ \\
\hline Physical activity & $1.07(0.91-1.22)$ & $1.18(1.00-1.36)^{*}$ & $1.35(1.06-1.64)^{*}$ & $1.39(1.16-1.61)^{* *}$ & $1.55(1.32-1.78) * * *$ & $1.97(1.57-2.38) * * *$ \\
\hline
\end{tabular}




\section{FIGURE LEGEND}

Figure 1:

50-64 years group: (1) "very low/decline", (2) "low/stable", (3) "average/stable", (4) "good/stable"; 65-79 years group: (1) "very low/rapid decline", (2) "low/decline", (3) "average/stable", (4) "good/stable".

\section{SUPPLEMENTARY MATERIAL}

Supplementary Figure S1. Drop-out probability within trajectory groups.

Supplementary Figure S2. Trajectories of verbal episodic memory by age group using model with drop-out extension. 